\title{
What factors are associated with recent intimate partner violence? findings from the WHO multi-country study on women's health and domestic violence
}

Tanya Abramsky ${ }^{1 *}$, Charlotte H Watts ${ }^{1}$, Claudia Garcia-Moreno ${ }^{2}$, Karen Devries ${ }^{1}$, Ligia Kiss ${ }^{1}$, Mary Ellsberg ${ }^{3}$, Henrica AFM Jansen ${ }^{4}$, Lori Heise ${ }^{1}$

\begin{abstract}
Background: Intimate partner violence (IPV) against women is a global public health and human rights concern. Despite a growing body of research into risk factors for IPV, methodological differences limit the extent to which comparisons can be made between studies. We used data from ten countries included in the WHO Multi-country Study on Women's Health and Domestic Violence to identify factors that are consistently associated with abuse across sites, in order to inform the design of IPV prevention programs.

Methods: Standardised population-based household surveys were done between 2000 and 2003. One woman aged 15-49 years was randomly selected from each sampled household. Those who had ever had a male partner were asked about their experiences of physically and sexually violent acts. We performed multivariate logistic regression to identify predictors of physical and/or sexual partner violence within the past 12 months.

Results: Despite wide variations in the prevalence of IPV, many factors affected IPV risk similarly across sites. Secondary education, high SES, and formal marriage offered protection, while alcohol abuse, cohabitation, young age, attitudes supportive of wife beating, having outside sexual partners, experiencing childhood abuse, growing up with domestic violence, and experiencing or perpetrating other forms of violence in adulthood, increased the risk of IPV. The strength of the association was greatest when both the woman and her partner had the risk factor.

Conclusions: IPV prevention programs should increase focus on transforming gender norms and attitudes, addressing childhood abuse, and reducing harmful drinking. Development initiatives to improve access to education for girls and boys may also have an important role in violence prevention.
\end{abstract}

\section{Background}

Intimate partner violence (IPV) against women is a global human rights and public health concern. The WHO Multi-Country Study on Women's Health and Domestic Violence documented the widespread nature of IPV [1], with lifetime prevalence of physical and/or sexual partner violence among ever-partnered women in the fifteen sites surveyed ranging from $15 \%$ in Ethiopia province to $71 \%$ in Japan. Between $4 \%-54 \%$ of respondents reported

\footnotetext{
* Correspondence: tanya.abramsky@lshtm.ac.uk

'London School of Hygiene and Tropical Medicine, 15-17 Tavistock Place, London WC1H 9SH, UK

Full list of author information is available at the end of the article
}

experiencing this violence in the year prior to the survey. In addition to being a concern in its own right, IPV is associated with a range of adverse physical, mental, sexual and reproductive health outcomes [2-8].

Designing effective IPV prevention programmes involves identification of risk factors-both those that are direct causes of IPV, and those that point to common characteristics of victims and/or perpetrators thus allowing appropriate tailoring and targeting of services. Studies in various countries have identified a range of factors that influence IPV risk [9-13], but in some cases, protective factors in one setting may be ineffective or actually increase risk in another [14]. For the purposes
C Biomed Central

() 2011 Abramsky et al; licensee BioMed Central Ltd. This is an Open Access article distributed under the terms of the Creative Commons Attribution License (http://creativecommons.org/licenses/by/2.0), which permits unrestricted use, distribution, and reproduction in any medium, provided the original work is properly cited. 
of intervention development, there is considerable interest in identifying a set of risk and protective factors for IPV that behave consistently across settings, to maximise chances of intervention success and minimise chances of inadvertently doing harm.

It is difficult to make comparisons between settings using existing individual studies as differences in identified risk factors may either be methodological artefacts or a real reflection of contrasting phenomena. Selected Demographic and Health Surveys [12,15] have added a Domestic Violence Module; however, country-level adaptations to the module and interviewer training procedures still limit their comparability. Standardisation is very important in a research field where even individual interviewer effects have a profound effect on level of disclosure [16].

We use population-based data from the WHO MultiCountry Study on Women's Health and Domestic Violence, which was specifically designed to better understand the factors associated with violence in different settings. Comparability of data was maximised through use of a standardised questionnaire, standardised interviewer training and data-collection procedures across all participating sites, and a rigorous set of quality control procedures. We drew on current models of IPV risk, including those of Heise $[17,18]$ and Jewkes [19] to develop a 'relationship' approach to assessing IPV risk. The characteristics and experiences of both the victim and the perpetrator are considered - in terms of what happened to each before they entered into the relationship, and their relative situations within the relationship alongside features and dynamics of the relationship itself (See Figure 1). Our goal is to identify factors that appear to consistently increase or decrease risk of partner violence across settings, and to identify where there are differences in patterns of association between sites.

\section{Methods}

Details of the study methods, sampling, response rates, and prevalence of different types of partner violence in each setting have been reported elsewhere [1] (see Additional file 1). Briefly, population-based surveys were conducted in Bangladesh, Brazil, Ethiopia, Japan, Namibia, Peru, Republic of Tanzania, Samoa, Serbia and Montenegro, and Thailand. In five countries surveys were done in the capital or another large city and one predominantly rural province. In the other five countries, only one site was surveyed because of logistical and financial considerations.

Trained female interviewers completed interviews with one randomly selected woman aged 15-49 from each sampled household. 24,097 women were interviewed in total, using a standardised questionnaire which was developed by the study team and translated into 14 languages.
Specially developed ethical guidelines emphasised the importance of ensuring confidentiality and privacy, both to protect the safety of respondents and field staff and to improve the quality of the data [20]. Ethical approval for the study was obtained from WHO's ethical review group (WHO Secretariat Committee for Research in Human Subjects), from the local institutions and, where necessary, national ethical review boards.

\section{Measures and Data Analysis IPV Outcome}

Currently- or previously-partnered women were asked a series of questions about whether they had ever experienced specific violent acts (see Figure 2), and if so whether this had happened in the 12 months preceding the survey. ${ }^{1}$ The analysis compared women who reported having experienced any act of physical and/or sexual violence in the past year (conceptualised as 'current violence') with women who did not report any partner violence ('current' versus 'never'). Those who had experienced partner violence during their lifetime but not in the past year were excluded from the analysis so as not to dilute associations.

\section{Risk-factors for IPV}

Variables are outlined in Figure 1, and consist of the woman's past history, her current/most recent partner's past history, the current situation of each of them (including relative status measures) and characteristics of the relationship and household. Variables were conceptualised as 'prior to relationship' if they preceded the relationship or 'current situation' if they related to the current situation within the relationship.

\section{Analysis Strategy}

All analyses were conducted separately by study site, using STATA 10.0. We used bivariate logistic regression to estimate the crude associations between each exposure variable and IPV, and to select variables for the multivariate analysis. Multivariate logistic regression was then used to model factors associated with past year IPV, separately for 'prior to relationship' characteristics, and 'current situation' variables. This chronological separation allowed us to explore the effects of the earlylife exposures independently of the later-life variables, which may be on the causal pathway (and thus attenuate the associations) between early-life experiences and later IPV. Clustering of outcomes in each site was 'small' (all intra-class correlation coefficients were less than 0.06) [21]; hence we present results unadjusted for clustering.

We included the same variables (decided upon $a$ priori) across the site-models except for the addition of certain relationship characteristics (polygamy and bride price/dowry) that were only relevant in some sites. As 


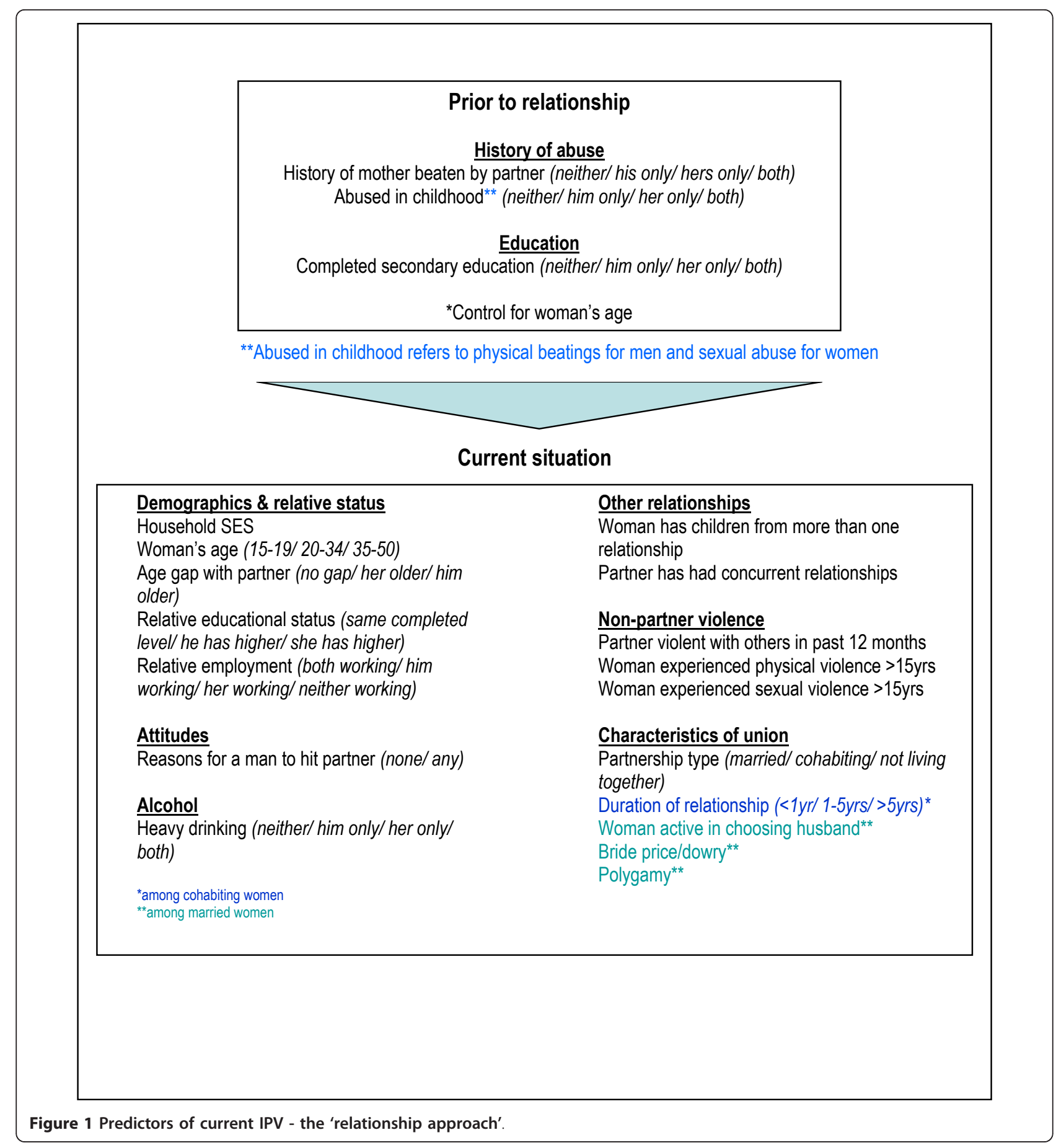

our overall aim was to identify similarities and differences in patterns of association between settings, we did not attempt to fit the most parsimonious model for each site. Neither did we place too much emphasis on the statistical significance of individual associations. Instead we focused on exploring the extent to which, keeping all other features of the model constant, patterns of associations were similar or different between sites.

When reporting results, we consider odds ratios (OR) between $0.95>\mathrm{OR}<1.05$ as indicative of no association, ORs of 1.05 or greater as risk-factors for IPV, and ORs of 0.95 or less as protective-factors for IPV. We use the terms risk- and protective-factors loosely to 


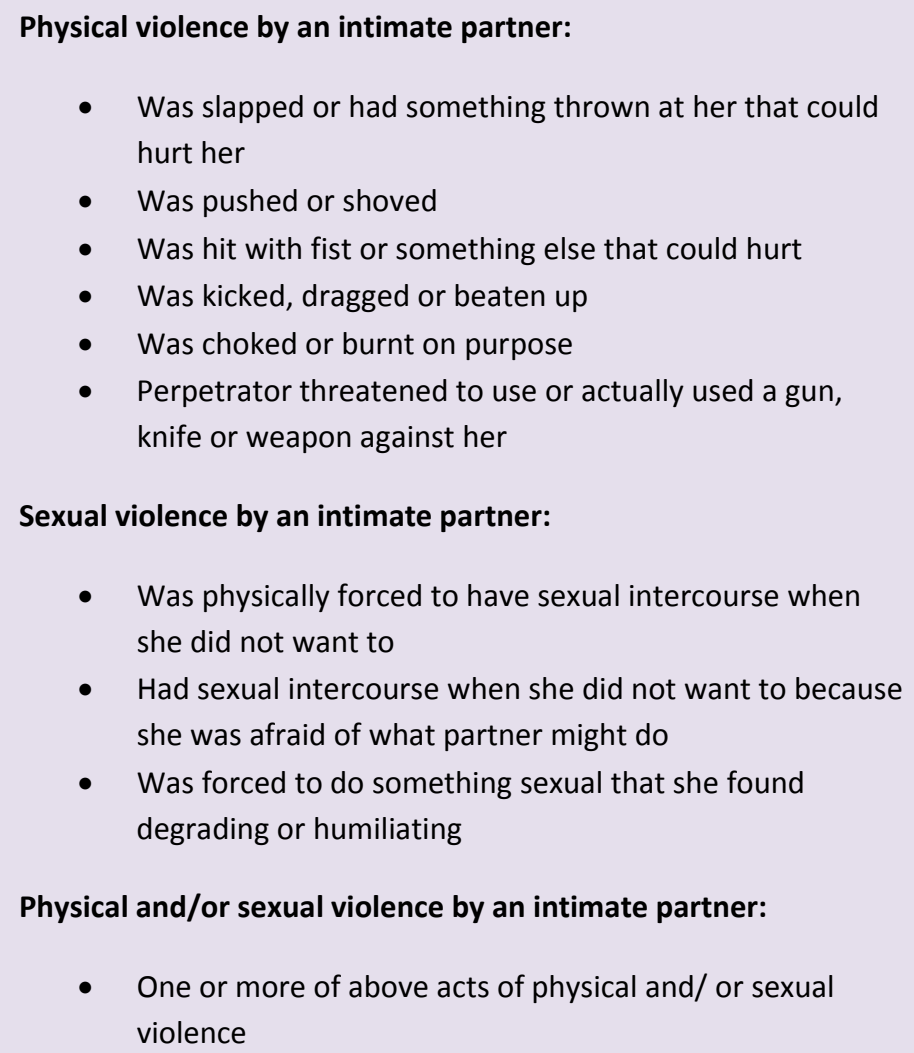

- Was slapped or had something thrown at her that could hurt her

- Was pushed or shoved

- Was hit with fist or something else that could hurt

- Was kicked, dragged or beaten up

- Was choked or burnt on purpose

- Perpetrator threatened to use or actually used a gun, knife or weapon against her

Sexual violence by an intimate partner:

- Was physically forced to have sexual intercourse when she did not want to

- Had sexual intercourse when she did not want to because she was afraid of what partner might do

- Was forced to do something sexual that she found degrading or humiliating

Physical and/or sexual violence by an intimate partner:

- One or more of above acts of physical and/ or sexual violence

Figure 2 Operational definitions of intimate partner violence.

indicate the direction of association with IPV rather than to imply causality, as we are analysing cross-sectional data. Statistical significance is considered at the $5 \%$ level.

\section{Results}

19,517 women reported having ever had a partner and were thus asked about partner violence. In total, having excluded women reporting lifetime but not past-year experience of violence, and those with missing data for key variables in the models, 15,207 women were included in the 'prior to relationship' analyses, and 15,058 in the 'current situation' analyses (see Tables 1 and 2).

Percentage distributions and adjusted odds ratios for all variables in the multivariate models are presented in Table 1 (prior to relationship), and Tables 2 and 3 (current relationship).

\section{Prior to relationship \\ Education}

Bivariate analysis of educational level indicated a reduction in IPV risk associated with secondary education for both the woman and her partner, but showed less consistent evidence of a protective effect of primary education. Therefore, when considering the woman and her partners' education in combination, we focused on complete versus incomplete secondary education (except for Bangladesh, Ethiopia and Tanzania where we examined primary completion because of extremely low secondary school enrolment). Achieving secondary education (or primary for Bangladesh, Ethiopia and Tanzania) by either the woman or her partner was associated with decreased IPV in almost two thirds of the sites (3 significant for each partner), when compared to situations where neither the woman nor her partner completed the level. However, the most consistent protective effect against IPV was observed where both the woman and her partner had completed the relevant schooling level (decreased risk in 12/14 sites, 10 significant). This most highly educated exposure group also had the lowest ORs for IPV in 10 out of 14 sites, compared to couples where one or both had not completed the level.

\section{History of abuse}

A history of abuse was strongly associated with the occurrence of IPV, with reports of abuse of the woman's 
Table 1 Descriptive data for 'prior to relationship' variables, and AORs* and 95\%Cls for associations with current IPV among ever partnered women

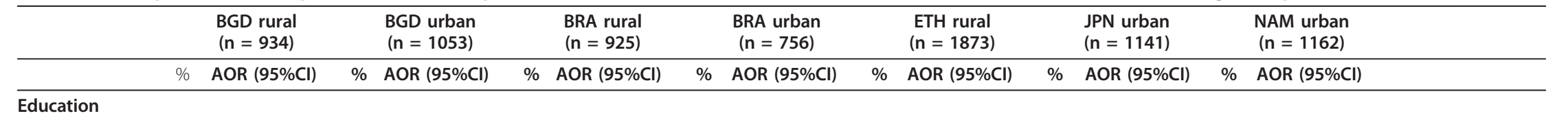

\section{Education}

Secondary completion**

\begin{tabular}{|c|c|c|c|c|c|c|c|c|c|c|c|c|c|c|c|c|}
\hline Neither completed & 38 & - & 11 & & 64 & - & 41 & - & 70 & - & 1 & - & 36 & - & & \\
\hline He completed & 22 & $0.75(0.52-1.1)$ & 13 & $0.45(0.26-0.78)$ & 5 & $0.76(0.33-1.7)$ & 10 & $0.37(0.15-0.92)$ & 7 & $1.1(0.73-1.6)$ & 2 & - & 16 & $0.74(0.49-1.1)$ & & \\
\hline She completed & 7 & $0.88(0.50-1.6)$ & 4 & $1.0(0.47-2.3)$ & 13 & $1.0(0.60-1.7)$ & 11 & $0.39(0.16-0.91)$ & 2 & $1.6(0.66-3.8)$ & 6 & - & 7 & $0.50(0.27-0.90)$ & & \\
\hline Both completed & 34 & $0.57(0.41-0.80)$ & 71 & $0.28(0.18-0.43)$ & 9 & $0.38(0.16-0.91)$ & 32 & $0.40(0.22-0.74)$ & 2 & $0.44(0.21-0.92)$ & 91 & - & 28 & $0.33(0.22-0.49)$ & & \\
\hline Missing data & 0 & - & 0 & - & 10 & $1.55(0.91-2.7)$ & 5 & $0.51(0.15-1.8)$ & 20 & $1.1(0.88-1.5)$ & 0 & - & 13 & $0.70(0.44-1.1)$ & & \\
\hline \multicolumn{17}{|l|}{ History of abuse } \\
\hline \multicolumn{17}{|c|}{ Reported history of mother abused } \\
\hline Neither mother & 81 & - & 79 & - & 69 & - & 69 & - & 74 & - & 80 & - & 76 & - & & \\
\hline His mother & 10 & $3.2(1.9-5.2)$ & 7 & $4.4(2.5-7.8)$ & 11 & $2.8(1.7-4.5)$ & 12 & $1.9(0.95-3.8)$ & 2 & $2.4(1.0-5.8)$ & 5 & $1.6(0.52-4.7)$ & 3 & $4.7(2.2-9.8)$ & & \\
\hline Her mother & 7 & $2.7(1.6-4.7)$ & 11 & $3.4(2.2-5.3)$ & 15 & $1.6(1.0-2.6)$ & 15 & $2.3(1.3-4.2)$ & 20 & $2.4(1.9-3.2)$ & 13 & $1.8(0.84-3.8)$ & 19 & $3.0(2.1-4.1)$ & & \\
\hline Both & 2 & $13.2(3.0-58.5)$ & 3 & $3.9(1.8-8.4)$ & 4 & $4.1(2.0-8.2)$ & 5 & $3.7(1.6-8.6)$ & 4 & $2.5(1.1-5.5)$ & 2 & $0.47(0.06-4.0)$ & 2 & $2.2(0.93-5.3)$ & & \\
\hline \multicolumn{17}{|c|}{ History of abuse as child } \\
\hline Neither & 90 & - & 83 & - & 78 & - & 74 & - & 96 & - & 88 & - & 90 & - & & \\
\hline $\mathrm{Him}$ & 9 & $2.4(1.4-4.2)$ & 11 & $1.6(1.1-2.5)$ & 17 & $1.9(1.2-2.9)$ & 20 & $1.7(0.98-3.1)$ & 4 & $2.9(1.2-7.0)$ & 4 & $6.6(2.4-18.2)$ & 6 & $1.8(1.0-3.1)$ & & \\
\hline Her & 1 & $2.5(0.59-10.6)$ & 5 & $2.5(1.3-4.7)$ & 3 & $4.5(2.1-9.6)$ & 5 & $2.9(1.2-7.1)$ & 0.1 & - & 8 & $5.3(2.6-10.8)$ & 4 & $1.6(0.80-3.3)$ & & \\
\hline \multirow[t]{3}{*}{ Both } & 0.3 & $1.6(0.13-20.7)$ & 1 & $15.2(1.9-123.8)$ & 2 & $3.4(1.2-10.1)$ & 2 & $5.8(1.9-17.9)$ & 0 & - & 1 & $15.1(3.3-68.5)$ & 0.4 & $0.69(0.07-6.6)$ & & \\
\hline & \multicolumn{2}{|c|}{ PER rural $(n=1008)$} & \multicolumn{2}{|c|}{ PER urban $(n=746)$} & \multicolumn{2}{|r|}{ SMA $(n=932)$} & \multicolumn{2}{|c|}{ SRB urban $(n=957)$} & \multicolumn{2}{|c|}{ THA rural $(n=781)$} & \multicolumn{2}{|c|}{ THA urban $(n=848)$} & \multicolumn{2}{|c|}{ TZA rural $(n=922)$} & & $\begin{array}{l}\text { TZA urban } \\
(\mathrm{n}=1169)\end{array}$ \\
\hline & $\%$ & AOR $(95 \% \mathrm{Cl})$ & $\%$ & AOR $(95 \% \mathrm{Cl})$ & $\%$ & AOR $(95 \% \mathrm{Cl})$ & $\%$ & AOR $(95 \% \mathrm{Cl})$ & $\%$ & AOR $(95 \% \mathrm{Cl})$ & $\%$ & AOR $(95 \% \mathrm{Cl})$ & $\%$ & AOR $(95 \% \mathrm{Cl})$ & $\%$ & AOR $(95 \% \mathrm{Cl})$ \\
\hline \multicolumn{17}{|l|}{ Education } \\
\hline \multicolumn{17}{|c|}{ Secondary completion** } \\
\hline Neither completed & 50 & - & 10 & - & 43 & - & 3 & - & 66 & - & 37 & - & 13 & - & 7 & - \\
\hline He completed & 21 & $0.93(0.66-1.3)$ & 15 & $1.5(0.76-2.8)$ & 13 & $0.68(0.42-1.1)$ & 4 & $1.1(0.26-4.8)$ & 7 & $0.86(0.46-1.6)$ & 14 & $1.3(0.80-2.1)$ & 19 & $0.47(0.28-0.78)$ & 15 & $1.4(0.68-2.7)$ \\
\hline She completed & 3 & $0.79(0.34-1.8)$ & 5 & $1.8(0.74-4.4)$ & 6 & $0.69(0.34-1.4)$ & 3 & $0.88(0.18-4.4)$ & 5 & $0.46(0.20-1.1)$ & 6 & $0.78(0.37-1.6)$ & 9 & $0.50(0.27-0.93)$ & 7 & $1.1(0.50-2.5)$ \\
\hline Both completed & 23 & $0.51(0.36-0.71)$ & 68 & $0.65(0.37-1.2)$ & 10 & $0.59(0.33-1.0)$ & 87 & $0.22(0.06-0.75)$ & 13 & $0.63(0.37-1.1)$ & 34 & $0.96(0.66-1.4)$ & 57 & $0.32(0.20-0.51)$ & 64 & $1.4(0.73-2.6)$ \\
\hline Missing data & 3 & $3.0(1.3-6.9)$ & 2 & $3.0(0.90-9.7)$ & 28 & $1.1(0.78-1.6)$ & 3 & $1.1(0.22-5.9)$ & 9 & $1.2(0.68-2.1)$ & 10 & $0.81(0.43-1.5)$ & 3 & $0.33(0.13-0.83)$ & 8 & $1.4(0.68-3.1)$ \\
\hline
\end{tabular}


Table 1 Descriptive data for ?'?prior to relationship?'? variables, and AORs* and 95\%Cls for associations with current IPV among ever partnered women (Continued)

\begin{tabular}{|c|c|c|c|c|c|c|c|c|c|c|c|c|c|c|c|c|}
\hline \multicolumn{17}{|l|}{ History of abuse } \\
\hline \multicolumn{17}{|c|}{ Reported history of mother abused } \\
\hline Neither mother & 34 & - & 49 & - & 54 & - & 80 & - & 65 & - & 69 & - & 47 & - & 68 & - \\
\hline His mother & 15 & $2.2(1.4-3.3)$ & 15 & $2.5(1.4-4.2)$ & 4 & $2.7(1.4-5.5)$ & 6 & $1.3(0.42-4.3)$ & 7 & $1.2(0.63-2.3)$ & 5 & $0.75(0.34-1.6)$ & 6 & $3.4(1.8-6.3)$ & 3 & $2.5(1.2-5.1)$ \\
\hline Her mother & 28 & $1.7(1.2-2.4)$ & 23 & $2.3(1.5-3.7)$ & 36 & $1.8(1.3-2.5)$ & 11 & $1.6(0.64-4.0)$ & 23 & $1.7(1.2-2.5)$ & 23 & $1.4(1.0-2.1)$ & 34 & $1.8(1.3-2.4)$ & 25 & $2.0(1.5-2.8)$ \\
\hline Both & 22 & $2.9(2.0-4.2)$ & 14 & $3.1(1.8-5.2)$ & 6 & $4.7(2.6-8.5)$ & 3 & $0.69(0.12-4.0)$ & 6 & $2.5(1.3-4.8)$ & 3 & $3.1(1.3-7.6)$ & 13 & $2.3(1.5-3.6)$ & 4 & $3.1(1.7-5.8)$ \\
\hline \multicolumn{17}{|c|}{ History of abuse as child } \\
\hline Neither & 61 & - & 58 & - & 91 & - & 93 & - & 85 & - & 86 & - & 89 & - & 91 & - \\
\hline Him & 32 & $1.5(1.1-2.1)$ & 25 & $2.2(1.4-3.3)$ & 7 & $2.9(1.7-4.9)$ & 6 & $9.5(3.9-23.4)$ & 11 & $3.0(1.9-4.9)$ & 8 & $3.2(1.9-5.6)$ & 7 & $2.5(1.4-4.3)$ & 5 & $1.6(0.89-2.8)$ \\
\hline$\overline{\mathrm{Her}}$ & 4 & $2.4(1.2-7.8)$ & 9 & $2.2(1.2-3.9)$ & 1 & $1.4(0.45-4.6)$ & 1 & $2.8(0.41-18.5)$ & 3 & $3.8(1.6-9.4)$ & 5 & $1.9(1.0-3.7)$ & 4 & $1.7(0.82-3.5)$ & 3 & $0.72(0.32-1.7)$ \\
\hline Both & 3 & $3.6(1.5-8.6)$ & 8 & $6.3(3.3-12.2)$ & 1 & $7.0(0.62-80.6)$ & 0.3 & $10.3(0.72-147)$ & 1 & $23.9(2.9-200.1)$ & 1 & $3.9(0.82-18.3)$ & 1 & $1.3(0.19-9.2)$ & 1 & $2.8(0.63-12.1)$ \\
\hline
\end{tabular}

${ }^{*}$ Adjusted for all variables in Table and woman's age.

**For Bangladesh Urban \& Rural, Ethiopia Rural and Tanzania Urban \& Rural, this variable refers to completed primary education. 
Table 2 Descriptive data for 'current situation' in relationship variables, and AORs* and 95\%Cls for associations with current IPV among ever partnered women

\begin{tabular}{|c|c|c|c|c|c|c|c|c|c|c|c|c|c|c|}
\hline & \multicolumn{2}{|c|}{ BGD rural $(n=926)$} & \multicolumn{2}{|c|}{$\begin{array}{l}\text { BGD urban }(n= \\
1050)\end{array}$} & \multicolumn{2}{|c|}{ BRA rural $(n=919)$} & \multicolumn{2}{|c|}{$\begin{array}{l}\text { BRA urban }(n= \\
746)\end{array}$} & \multicolumn{2}{|c|}{ ETH rural $(n=1861)$} & \multicolumn{2}{|c|}{$\underset{1075)}{J P N}$ urban $(n=$} & \multicolumn{2}{|c|}{$\begin{array}{c}\text { NAM urban } \\
1154)\end{array}$} \\
\hline & $\%$ & AOR $(95 \% \mathrm{Cl})$ & $\%$ & AOR $(95 \% \mathrm{Cl})$ & $\%$ & AOR $(95 \% \mathrm{Cl})$ & $\%$ & AOR $(95 \% \mathrm{Cl})$ & $\%$ & AOR $(95 \% \mathrm{Cl})$ & $\%$ & AOR $(95 \% \mathrm{Cl})$ & $\%$ & AOR $(95 \% \mathrm{Cl})$ \\
\hline \multicolumn{15}{|l|}{ Demographics } \\
\hline \multicolumn{15}{|l|}{ SES } \\
\hline Low & 76 & - & 72 & - & 26 & - & 34 & - & 84 & - & 30 & - & 27 & - \\
\hline Medium & 20 & $0.71(0.49-1.0)$ & 22 & $0.68(0.48-0.96)$ & 64 & $0.63(0.40-0.99)$ & 40 & $0.99(0.54-1.8)$ & 14 & $0.91(0.66-1.3)$ & 23 & $0.71(0.33-1.5)$ & 26 & $1.3(0.81-2.0)$ \\
\hline High & 4 & $0.69(0.34-1.4)$ & 7 & $0.33(0.17-0.66)$ & 11 & $0.75(0.35-1.6)$ & 26 & $0.25(0.09-0.67)$ & 2 & $0.53(0.25-1.1)$ & 47 & $0.24(0.10-0.58)$ & 47 & $0.94(0.61-1.5)$ \\
\hline \multicolumn{15}{|l|}{ Woman's age } \\
\hline $35+$ & 35 & - & 28 & - & 39 & - & 46 & - & 41 & - & 54 & - & 36 & - \\
\hline $20-35$ yrs & 57 & $2.1(1.5-3.1)$ & 64 & $2.7(1.8-4.0)$ & 54 & $1.7(1.0-2.7)$ & 47 & $2.4(1.2-4.6)$ & 55 & $2.4(1.9-3.1)$ & 44 & $0.78(0.38-1.62)$ & 58 & $0.93(0.62-1.4)$ \\
\hline $15-19$ yrs & 8 & $3.2(1.7-6.2)$ & 9 & $4.1(2.2-7.7)$ & 8 & $2.9(1.2-6.7)$ & 6 & $4.2(1.3-13.1)$ & 4 & $1.7(0.97-3.1)$ & 2 & $1.1(0.09-13.6)$ & 6 & $1.1(0.47-2.4)$ \\
\hline \multicolumn{15}{|c|}{ Age gap with partner (> = $5 \mathrm{yrs}$ ) } \\
\hline None & 11 & - & 16 & - & 52 & - & 58 & - & 11 & - & 75 & - & 55 & - \\
\hline $\begin{array}{c}\text { She is }>=5 \\
\text { yrs older }\end{array}$ & 0 & - & 0.1 & - & 5 & $0.89(0.33-2.4)$ & 6 & $1.3(0.42-3.8)$ & 1 & $1.1(0.31-3.6)$ & 2 & $0.65(0.07-6.0)$ & 2 & $1.2(0.46-3.2)$ \\
\hline $\begin{array}{l}\text { He is }>=5 \\
\text { yrs older }\end{array}$ & 89 & $0.90(0.56-1.44)$ & 84 & $0.81(0.56-1.2)$ & 43 & $0.99(0.67-1.5)$ & 36 & $0.89(0.50-1.6)$ & 88 & $1.3(0.93-1.7)$ & 24 & $0.92(0.42-2.0)$ & 43 & $0.96(0.69-1.34)$ \\
\hline \multicolumn{15}{|c|}{ Relative education } \\
\hline Same level & 64 & - & 61 & - & 60 & - & 61 & - & 71 & - & 92 & - & 54 & - \\
\hline $\begin{array}{l}\text { He has } \\
\text { higher }\end{array}$ & 28 & $0.74(0.50-1.09)$ & 34 & $1.1(0.84-1.6)$ & 11 & $1.6(0.83-2.9)$ & 17 & $0.73(0.35-1.6)$ & 8 & $1.0(0.50-2.1)$ & 2 & $1.6(0.33-8.3)$ & 22 & $1.2(0.80-1.8)$ \\
\hline $\begin{array}{l}\text { She has } \\
\text { higher }\end{array}$ & 7 & $0.83(0.58-1.19)$ & 5 & $1.1(0.74-1.7)$ & 19 & $1.6(0.97-2.7)$ & 18 & $1.1(0.53-2.1)$ & 2 & $1.3(0.82-1.9)$ & 6 & $0.94(0.25-3.6)$ & 12 & $1.0(0.63-1.7)$ \\
\hline Missing & - & - & - & - & 10 & $1.5(0.80-2.7)$ & 5 & $0.1(0.02-0.50)$ & 20 & $1.4(1.1-1.8)$ & - & - & 13 & $0.98(0.58-1.6)$ \\
\hline \multicolumn{15}{|c|}{ Relative employment } \\
\hline Both working & 18 & - & 15 & - & 31 & - & 56 & - & 36 & - & 53 & - & 48 & - \\
\hline Man working & 74 & $0.58(0.40-0.83)$ & 80 & $0.88(0.60-1.3)$ & 52 & $0.83(0.52-1.3)$ & 31 & $1.3(0.70-2.4)$ & 62 & $0.99(0.79-1.2)$ & 40 & $1.4(0.7-3.0)$ & 35 & $1.19(0.82-1.7)$ \\
\hline $\begin{array}{l}\text { Woman } \\
\text { working }\end{array}$ & 2 & $1.0(0.40-2.6)$ & 1 & $0.72(0.10-5.2)$ & 7 & $1.0(0.48-2.2)$ & 8 & $2.8(1.2-6.7)$ & 1 & $0.75(0.26-2.1)$ & 5 & $6.0(1.5-23.3)$ & 7 & $0.83(0.43-1.6)$ \\
\hline $\begin{array}{l}\text { Neither } \\
\text { working }\end{array}$ & 6 & $0.99(0.52-1.9)$ & 4 & $0.75(0.33-1.7)$ & 10 & $1.2(0.63-2.4)$ & 5 & $1.1(0.36-3.3)$ & 1 & $0.54(0.20-1.5)$ & 2 & No cases & 10 & $1.9(1.1-3.2)$ \\
\hline \multicolumn{15}{|l|}{ Attitudes } \\
\hline \multicolumn{15}{|c|}{ Reasons for a man to hit partner } \\
\hline None & 20 & - & 47 & - & 69 & - & 90 & - & 8 & - & 82 & - & 79 & - \\
\hline Any & 80 & $2.5(1.7-3.6)$ & 53 & $1.9(1.4-2.5)$ & 31 & $1.5(1.0-2.2)$ & 10 & $2.8(1.4-5.8)$ & 92 & $1.6(1.1-2.2)$ & 18 & $1.7(0.82-3.4)$ & 21 & $1.4(0.99-2.1)$ \\
\hline
\end{tabular}


Table 2 Descriptive data for 'current situation' in relationship variables, and AORs* and 95\%Cls for associations with current IPV among ever partnered women (Continued)

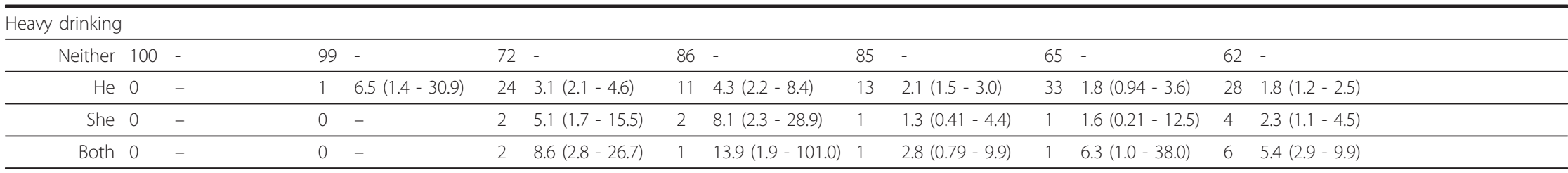

Non-intimate-partner violence

Victim of sexual abuse (>15 yrs)

\begin{tabular}{|c|c|c|c|c|c|c|c|c|c|c|c|c|c|c|}
\hline No & 99.6 & - & 94 & - & 96 & - & 94 & - & 99.7 & - & 97 & - & 95 & - \\
\hline Yes & 0.4 & - & 6 & $1.2(0.67-2.2)$ & 4 & $2.2(0.92-5.2)$ & 6 & $2.3(0.97-5.6)$ & 0.3 & - & 3 & $7.1(2.2-22.5)$ & 5 & $7.3(3.7-14.1)$ \\
\hline \multicolumn{15}{|c|}{ Victim of physical abuse (>15 yrs) } \\
\hline No & 93 & - & 86 & - & 88 & - & 80 & - & 96 & - & 96 & - & 82 & - \\
\hline Yes & 7 & $1.6(0.93-2.8)$ & 14 & $1.3(0.86-1.9)$ & 12 & $1.8(1.1-3.1)$ & 20 & $2.1(1.1-3.9)$ & 4 & $2.6(1.4-5.0)$ & 4 & $5.4(2.0-14.4)$ & 18 & $2.4(1.6-3.4)$ \\
\hline \multicolumn{15}{|c|}{ Partner has fought with other man (past yr) } \\
\hline No & 96 & - & 96 & - & 92 & - & 91 & - & 89 & - & 97 & - & 90 & - \\
\hline Yes & 4 & $5.7(2.3-14.3)$ & 4 & $4.5(1.9-10.5)$ & 8 & $4.1(2.3-7.2)$ & 9 & $3.2(1.6-6.7)$ & 11 & $4.2(2.7-6.7)$ & 3 & $11.3(3.7-34.3)$ & 10 & $2.5(1.5-4.0)$ \\
\hline
\end{tabular}

Other relationships

Woman has children from $>1$ father

\begin{tabular}{|c|c|c|c|c|c|c|c|c|c|c|c|c|c|c|c|c|}
\hline $\begin{array}{r}1 \text { father }(>1 \\
\text { kid) }\end{array}$ & 77 & - & 63 & - & 53 & - & 43 & - & 78 & - & 71 & - & 28 & - & & \\
\hline$>1$ father & 1 & $2.2(0.66-7.4)$ & 1 & $4.9(0.76-31.2)$ & 18 & $1.1(0.61-1.8)$ & 11 & $2.2(0.88-5.5)$ & 10 & $0.99(0.68-1.4))$ & 1 & - & 30 & $1.4(0.91-2.3)$ & & \\
\hline 0-1 kids & 21 & $0.59(0.38-0.94)$ & 36 & $0.92(0.64-1.3)$ & 30 & $0.85(0.48-1.5)$ & 46 & $0.98(0.46-2.1)$ & 12 & $0.98(0.63-1.5)$ & 28 & - & 42 & $1.4(0.85-2.3)$ & & \\
\hline \multicolumn{17}{|c|}{ Partner has had concurrent relationship(s) } \\
\hline No & 95 & - & 94 & - & 69 & - & 81 & - & 73 & - & 91 & - & 57 & - & & \\
\hline Yes & 3 & $5.8(1.6-21.7)$ & 3 & $7.5(2.4-23.1)$ & 25 & $2.1(1.4-3.1)$ & 13 & $4.5(2.3-8.8)$ & 27 & $1.2(0.97-1.6)$ & 3 & $5.8(1.7-19.8)$ & 23 & $1.7(1.2-2.4)$ & & \\
\hline DK/maybe & 3 & $3.4(1.3-9.2)$ & 3 & $2.1(0.85-5.1)$ & 6 & $1.2(0.52-2.6)$ & 6 & $2.7(1.0-7.0)$ & 0.3 & $0.52(0.10-2.8)$ & 6 & $0.63(0.15-2.6)$ & 19 & $0.97(0.6-1.5)$ & & \\
\hline \multicolumn{17}{|c|}{ Characteristics of union } \\
\hline \multicolumn{17}{|c|}{ Any marriage ceremony } \\
\hline Married & 50 & - & 78 & - & 48 & - & 56 & - & 98 & - & 87 & - & 36 & - & & \\
\hline $\begin{array}{l}\text { No, living } \\
\text { together }\end{array}$ & 50 & $0.88(0.66-1.2)$ & 22 & $0.81(0.57-1.1)$ & 48 & $1.4(0.89-2.1)$ & 30 & $1.1(0.57-2.1)$ & 2 & $0.61(0.30-1.3)$ & 0.1 & - & 29 & $1.6(1.1-2.5)$ & & \\
\hline \multirow[t]{3}{*}{$\begin{array}{l}\text { Not living } \\
\text { together }\end{array}$} & - & - & - & - & 5 & $0.94(0.34-2.6)$ & 15 & $0.60(0.20-1.8)$ & 0.2 & - & 13 & $0.39(0.08-1.8)$ & 35 & $0.97(0.62-1.5)$ & & \\
\hline & \multicolumn{2}{|c|}{ PER rural $(n=1005)$} & \multicolumn{2}{|c|}{ PER urban $(n=736)$} & \multicolumn{2}{|r|}{ SMA $(n=931)$} & \multicolumn{2}{|c|}{ SRB urban $(n=954)$} & \multicolumn{2}{|c|}{ THA rural $(n=772)$} & \multicolumn{2}{|c|}{ THA urban $(n=845)$} & \multicolumn{2}{|c|}{ TZA rural $(n=918)$} & & $\begin{array}{l}\text { TZA urban } \\
(\mathrm{n}=1166)\end{array}$ \\
\hline & $\%$ & AOR $(95 \% \mathrm{Cl})$ & $\%$ & AOR $(95 \% \mathrm{Cl})$ & $\%$ & AOR $(95 \% \mathrm{Cl})$ & $\%$ & AOR $(95 \% \mathrm{Cl})$ & $\%$ & AOR $(95 \% \mathrm{Cl})$ & $\%$ & AOR $(95 \% \mathrm{Cl})$ & $\%$ & AOR $(95 \% \mathrm{Cl})$ & $\%$ & AOR $(95 \% \mathrm{Cl})$ \\
\hline
\end{tabular}


Table 2 Descriptive data for 'current situation' in relationship variables, and AORs* and $95 \%$ Cls for associations with current IPV among ever partnered women (Continued)

Demographics

SES

\begin{tabular}{|c|c|c|c|c|c|c|c|c|c|c|c|c|c|c|c|c|}
\hline Low & 47 & - & 11 & - & 15 & - & 18 & - & 9 & - & 10 & - & 86 & - & 65 & - \\
\hline Medium & 34 & $1.4(0.97-2.0)$ & 22 & $0.57(0.29-1.1)$ & 50 & $0.54(0.35-0.84)$ & 44 & $0.66(0.25-1.74)$ & 49 & $0.67(0.37-1.2)$ & 25 & $0.84(0.45-1.6)$ & 10 & $0.72(0.42-1.2)$ & 23 & $1.1(0.76-1.6)$ \\
\hline High & 19 & $0.61(0.36-1.0)$ & 66 & $0.56(0.30-1.0)$ & 36 & $0.34(0.21-0.56)$ & 38 & $0.41(0.15-1.18)$ & 42 & $0.45(0.24-0.85)$ & 65 & $0.74(0.41-1.3)$ & 4 & $1.1(0.51-2.5)$ & 12 & $1.3(0.81-2.2)$ \\
\hline \multicolumn{17}{|l|}{ Woman's age } \\
\hline $35+$ & 42 & - & 42 & - & 44 & - & 53 & - & 59 & - & 48 & - & 28 & - & 31 & - \\
\hline $20-35$ yrs & 54 & $1.4(0.94-2.0)$ & 52 & $4.6(2.6-8.0)$ & 53 & $1.6(1.1-2.4)$ & 44 & $2.04(0.82-5.1)$ & 38 & $1.1(0.74-1.6)$ & 49 & $1.8(1.2-2.6)$ & 63 & $1.9(1.2-2.9)$ & 60 & $2.0(1.3-3.0)$ \\
\hline $15-19$ yrs & 4 & $2.4(1.1-5.3)$ & 5 & $11.8(4.4-31.3)$ & 2 & $2.9(1.0-8.4)$ & 3 & $16.2(2.5-104.2)$ & 3 & $1.7(0.58-5.0)$ & 3 & $3.1(1.1-8.4)$ & 9 & $3.4(1.7-6.8)$ & 9 & $2.0(0.99-3.9)$ \\
\hline \multicolumn{17}{|c|}{ Age gap with partner ( $>=5$ yrs) } \\
\hline None & 61 & - & 58 & - & 54 & - & 66 & - & 64 & - & 59 & - & 38 & - & 33 & - \\
\hline $\begin{array}{r}\text { She is }>=5 \\
\text { yrs older }\end{array}$ & 2 & $0.84(0.26-2.8)$ & 4 & $1.8(0.67-5.0)$ & 4 & $1.9(0.84-4.1)$ & 1 & $13.3(1.5-115.5)$ & 3 & $1.2(0.46-3.1)$ & 3 & $0.47(0.14-1.6)$ & 0.3 & - & 0.1 & - \\
\hline $\begin{array}{r}\mathrm{He} \text { is }>=5 \\
\text { yrs older }\end{array}$ & 37 & $1.2(0.87-1.7)$ & 39 & $0.74(0.48-1.1)$ & 42 & $0.88(0.64-1.2)$ & 33 & $2.0(0.91-4.4)$ & 33 & $0.87(0.59-1.3)$ & 38 & $1.0(0.69-1.4)$ & 61 & $0.75(0.55-1.0)$ & 67 & $0.82(0.60-1.1)$ \\
\hline \multicolumn{17}{|c|}{ Relative education } \\
\hline Same level & 54 & - & 75 & - & 49 & - & 90 & - & 62 & - & 61 & - & 65 & - & 60 & - \\
\hline $\begin{array}{l}\text { He has } \\
\text { higher }\end{array}$ & 38 & $1.0(0.87-1.7)$ & 18 & $1.4(0.84-2.5)$ & 14 & $0.92(0.58-1.5)$ & 4 & $4.6(1.4-15.5)$ & 18 & $1.3(0.75-2.1)$ & 20 & $1.4(0.92-2.2)$ & 23 & $1.5(0.94-2.4)$ & 25 & $0.77(0.50-1.2)$ \\
\hline $\begin{array}{r}\text { She has } \\
\text { higher }\end{array}$ & 5 & $1.2(0.81-1.9)$ & 5 & $1.7(0.82-3.4)$ & 8 & $0.86(0.45-1.7)$ & 3 & $6.5(1.5-27.1)$ & 11 & $1.0(0.62-1.7)$ & 10 & $1.1(0.62-1.9)$ & 9 & $1.1(0.74-1.6)$ & 7 & $0.73(0.48-1.1)$ \\
\hline Missing & 3 & $3.4(1.3-9.3)$ & 2 & $6.3(1.8-22.7)$ & 28 & $1.1(0.74-1.6)$ & 3 & $4.0(1.0-15.3)$ & 9 & $1.5(0.80-2.8)$ & 10 & $0.72(0.37-1.4)$ & 3 & $1.1(0.43-2.9)$ & 8 & $1.0(0.55-2.0)$ \\
\hline
\end{tabular}

Relative employment

\begin{tabular}{|c|c|c|c|c|c|c|c|c|c|c|c|c|c|c|c|c|}
\hline Both working & 51 & - & 59 & - & 43 & - & - & - & 75 & - & 70 & - & 61 & - & 40 & - \\
\hline Man working & 37 & $1.1(0.79-1.5)$ & 26 & $0.79(0.49-1.3)$ & 43 & $0.89(0.63-1.3)$ & - & - & 17 & $0.88(0.55-1.4)$ & 23 & $0.65(0.41-1.0)$ & 33 & $1.1(0.80-1.5)$ & 46 & $0.91(0.66-1.2)$ \\
\hline $\begin{array}{l}\text { Woman } \\
\text { working }\end{array}$ & 8 & $1.1(0.63-2.0)$ & 11 & $1.8(0.99-3.4)$ & 7 & $0.71(0.35-1.4)$ & - & - & 6 & $0.83(0.39-1.8)$ & 5 & $1.5(0.74-3.2)$ & 3 & $1.4(0.55-3.6)$ & 6 & $1.0(0.55-2.0)$ \\
\hline $\begin{array}{l}\text { Neither } \\
\text { working }\end{array}$ & 3 & $2.2(0.89-5.3)$ & 4 & $1.2(0.38-3.5)$ & 7 & $1.2(0.62-2.2)$ & - & - & 3 & $2.0(0.72-5.7)$ & 2 & $6.4(1.9-21.5)$ & 3 & $0.92(0.38-2.2)$ & 8 & $0.72(0.39-1.3)$ \\
\hline \multicolumn{17}{|l|}{ Attitudes } \\
\hline \multicolumn{17}{|c|}{ Reasons for a man to hit partner } \\
\hline None & 19 & - & 67 & - & 28 & - & 95 & - & 29 & - & 52 & - & 33 & - & 37 & - \\
\hline Any & 81 & $1.5(0.94-2.3)$ & 33 & $1.4(0.92-2.1)$ & 72 & $1.5(1.0-2.1)$ & 5 & $1.3(0.32-5.0)$ & 71 & $0.95(0.64-1.4)$ & 48 & $0.95(0.67-1.3)$ & 67 & $1.8(1.3-2.5)$ & 63 & $1.9(1.3-2.6)$ \\
\hline
\end{tabular}

Alcohol use

Heavy drinking

Neither 51

$70-$

$67-$

$94-$

64

$73-$

$78 \quad-$ 
Table 2 Descriptive data for 'current situation' in relationship variables, and AORs* and 95\%Cls for associations with current IPV among ever partnered women (Continued)

\begin{tabular}{|c|c|c|c|c|c|c|c|c|c|c|c|c|c|c|c|c|}
\hline $\mathrm{He}$ & 31 & $3.3(2.3-4.6)$ & 19 & $2.5(1.5-4.1)$ & 32 & $1.5(1.1-2.1)$ & 6 & $8.6(3.5-21.3)$ & 26 & $2.7(1.9-4.0)$ & 21 & $2.1(1.4-3.1)$ & 18 & $3.4(2.3-5.1)$ & 19 & $2.5(1.8-3.6)$ \\
\hline She & 7 & $2.1(1.1-3.7)$ & 8 & $1.0(0.48-2.1)$ & 0.3 & - & 0.2 & - & 5 & $2.6(1.3-5.4)$ & 3 & $1.1(0.40-2.9)$ & 2 & $1.3(0.44-4.1)$ & 2 & $1.6(0.56-4.8)$ \\
\hline Both & 12 & $11.8(6.1-22.8)$ & 4 & $1.1(0.42-3.0)$ & 0.3 & - & 0 & - & 5 & $6.0(2.8-13.0)$ & 3 & $2.6(0.98-6.6)$ & 2 & $4.3(1.6-11.6)$ & 1 & $2.9(0.97-8.7)$ \\
\hline
\end{tabular}

Non-intimate-partner violence

Victim of sexual abuse (>15 yrs)

\begin{tabular}{|c|c|c|c|c|c|c|c|c|c|c|c|c|c|c|c|c|}
\hline No & 89 & - & 89 & - & 91 & - & 98 & - & 98 & - & 94 & - & 92 & - & 91 & \\
\hline Yes & 11 & $2.6(1.5-4.5)$ & 11 & $2.1(1.2-3.9)$ & 9 & $1.2(0.69-2.0)$ & 2 & $0.26(0.03-2.6)$ & 2 & $1.3(0.41-4.3)$ & 6 & $2.6(1.3-5.3)$ & 8 & $1.4(0.78-2.4)$ & 9 & $2.8(1.7-4.5)$ \\
\hline \multicolumn{17}{|c|}{ Victim of physical abuse (>15 yrs) } \\
\hline No & 68 & - & 72 & - & 41 & - & 92 & - & 91 & - & 93 & - & 86 & - & 83 & - \\
\hline Yes & 32 & $2.0(1.4-2.7)$ & 28 & $2.3(1.5-3.5)$ & 59 & $1.2(0.85-1.6)$ & 8 & $3.1(1.1-8.6)$ & 9 & $1.9(1.1-3.4)$ & 7 & $3.0(1.6-5.5)$ & 14 & $1.3(0.82-1.9)$ & 17 & $0.93(0.63-1.4)$ \\
\hline \multicolumn{17}{|c|}{ Partner has fought with other man (past yr) } \\
\hline No & 78 & - & 91 & - & 82 & - & 88 & - & 86 & - & 88 & - & 95 & - & 95 & - \\
\hline Yes & 22 & $2.3(1.5-3.6)$ & 9 & $2.5(1.3-4.7)$ & 18 & $3.7(2.5-5.4)$ & 12 & $4.6(2.0-10.6)$ & 14 & $2.6(1.6-4.1)$ & 12 & $3.6(2.2-5.8)$ & 5 & $1.9(0.84-4.2)$ & 5 & $1.7(0.89-3.2)$ \\
\hline \multicolumn{17}{|c|}{ Other relationships } \\
\hline \multicolumn{17}{|c|}{ Woman has children from $>1$ father } \\
\hline $\begin{array}{r}1 \text { father }(>1 \\
\text { kid) }\end{array}$ & 69 & - & 49 & - & 70 & - & 42 & - & 55 & - & 47 & - & 61 & - & 45 & - \\
\hline$>1$ father & 8 & $2.2(1.1-4.8)$ & 9 & $2.2(0.99-5.1)$ & 11 & $2.1(1.2-3.6)$ & 2 & $3.6(0.30-41.6)$ & 7 & $0.89(0.41-1.9)$ & 5 & $1.5(0.64-3.3)$ & 14 & $1.5(0.91-2.4)$ & 16 & $1.4(0.90-2.2)$ \\
\hline $0-1$ kids & 23 & $0.89(0.55-1.4)$ & 41 & $0.83(0.47-1.5)$ & 19 & $1.6(0.94-2.6)$ & 56 & $1.5(0.53-4.2)$ & 38 & $1.4(0.89-2.1)$ & 48 & $0.96(0.62-1.5)$ & 25 & $0.81(0.51-1.3)$ & 40 & $0.65(0.42-1.0)$ \\
\hline \multicolumn{17}{|c|}{ Partner has had concurrent relationship(s) } \\
\hline No & 67 & - & 69 & - & 81 & - & 87 & - & 72 & - & 66 & - & 61 & - & 53 & - \\
\hline Yes & 20 & $4.0(2.6-6.2)$ & 17 & $5.2(3.1-8.7)$ & 17 & $2.0(1.3-3.0)$ & 4 & $6.6(2.0-21.7)$ & 19 & $2.4(1.6-3.7)$ & 17 & $2.1(1.4-3.3)$ & 21 & $3.1(2.1-4.5)$ & 16 & $2.9(1.9-4.2)$ \\
\hline DK/maybe & 13 & $2.4(1.5-3.9)$ & 13 & $2.7(1.5-4.6)$ & 2 & - & 8 & $2.3(0.75-6.8)$ & 9 & $3.4(1.9-6.0)$ & 16 & $1.4(0.86-2.2)$ & 18 & $2.1(1.5-3.2)$ & 31 & $1.3(0.91-1.8)$ \\
\hline
\end{tabular}

Characteristics of union

Any marriage ceremony

\begin{tabular}{|c|c|c|c|c|c|c|c|c|c|c|c|c|c|c|c|c|}
\hline Married & 59 & - & 53 & - & 74 & - & 69 & - & 89 & - & 82 & - & 57 & - & 61 & - \\
\hline $\begin{array}{l}\text { No, living } \\
\text { together }\end{array}$ & 36 & $1.2(0.81-1.7)$ & 27 & $1.1(0.65-1.7)$ & 26 & $2.5(0.93-6.5)$ & 11 & $3.1(1.1-8.6)$ & 10 & $1.6(0.90-2.7)$ & 17 & $0.84(0.53-1.3)$ & 37 & $1.2(0.84-1.6)$ & 24 & $1.8(1.3-2.6)$ \\
\hline $\begin{array}{c}\text { Not living } \\
\text { together }\end{array}$ & 6 & $0.75(0.34-1.7)$ & 20 & $0.52(0.26-1.0)$ & 0 & - & 20 & $0.34(0.07-1.7)$ & 1 & - & 1 & - & 6 & $0.39(0.17-0.88)$ & 15 & $0.67(0.40-1.1)$ \\
\hline
\end{tabular}

*Adjusted for all variables in Table, and parity 
Table 3 Descriptive data for additional 'characteristics of union' variables, and AORs* and 95\%Cls for associations with current IPV among cohabiting and married women

\begin{tabular}{|c|c|c|c|c|c|c|c|c|c|c|c|c|c|c|c|c|}
\hline & & BGD rural & & BGD urban & & BRA rural & & BRA urban & & ETH rural & & JPN urban & & NAM urban & & \\
\hline & $\%$ & AOR $(95 \% \mathrm{Cl})$ & $\%$ & AOR $(95 \% \mathrm{Cl})$ & $\%$ & AOR $(95 \% \mathrm{Cl})$ & $\%$ & AOR $(95 \% \mathrm{Cl})$ & $\%$ & AOR $(95 \% \mathrm{Cl})$ & $\%$ & AOR $(95 \% \mathrm{Cl})$ & $\%$ & AOR $(95 \% \mathrm{Cl})$ & & \\
\hline Among cohabiting & & $(n=926)$ & & $(n=1050)$ & & $(n=870)$ & & $(n=639)$ & & $(n=1859)$ & & $(n=833)$ & & $(n=754)$ & & \\
\hline \multicolumn{17}{|c|}{ Duration of relationship } \\
\hline$>=5 \mathrm{yrs}$ & 80 & - & 75 & - & 68 & - & 68 & - & 85 & - & - & - & 61 & - & & \\
\hline $1-5$ yrs & 16 & $0.63(0.36-1.1)$ & 21 & $0.94(0.61-1.4)$ & 24 & $0.68(0.38-1.2)$ & 26 & $0.67(0.30-1.5)$ & 11 & $0.68(0.45-1.0)$ & - & - & 30 & $0.83(0.50-1.4)$ & & \\
\hline$<1 \mathrm{yr}$ & 4 & $0.57(0.21-1.5)$ & 3 & $0.44(0.17-1.1)$ & 7 & $1.3(0.60-3.0)$ & 6 & $0.86(0.23-3.3)$ & 3 & $0.49(0.24-0.97)$ & - & - & 9 & $0.95(0.43-2.1)$ & & \\
\hline Among cohabiting & & $(n=926)$ & & $(n=1046)$ & & $(n=870)$ & & $(n=639)$ & & $(n=1821)$ & & $(n=830)$ & & $(n=407)$ & & \\
\hline \multicolumn{17}{|l|}{ Choice of husband } \\
\hline She took part & 5 & - & 16 & - & 98 & - & 98 & - & 6 & - & 98 & - & 72 & - & & \\
\hline He/family chose & 95 & $0.66(0.34-1.3)$ & 84 & $0.63(0.43-0.93)$ & 2 & $1.0(0.17-6.4)$ & 2 & $6.3(1.3-31.7)$ & 94 & $1.6(1.0-2.4)$ & 2 & $3.2(0.51-19.6)$ & 28 & $0.62(0.28-1.4)$ & & \\
\hline Bride price/dowry & & (binary) & & (binary) & & & & & & & & & & & & \\
\hline Dowry & 54 & $1.8(1.3-2.4)$ & 12 & $2.3(1.5-3.5)$ & & & & & 10 & $1.4(1.0-2.1)$ & & & 9 & $1.7(0.57-5.0)$ & & \\
\hline Bride price & 1 & - & 1 & - & & & & & 7 & $0.65(0.45-0.95)$ & & & 19 & $0.78(0.32-1.9)$ & & \\
\hline None & 45 & - & 87 & - & & & & & 80 & - & & & 72 & - & & \\
\hline 4 & - & & - & & & & & & 2 & $0.57(0.30-1.1)$ & & & & & & \\
\hline \multicolumn{17}{|l|}{ Polygamy } \\
\hline No & 98 & - & 97 & - & & & & & 69 & - & & & 76 & - & & \\
\hline Yes & 2 & $1.66(0.58-4.7)$ & 3 & $3.0(1.2-7.1)$ & & & & & 31 & $1.3(0.83-2.0)$ & & & 11 & $2.6(1.0-6.6)$ & & \\
\hline \multirow[t]{3}{*}{ Don't know } & 0.3 & - & - & - & & & & & 0.1 & $1.1(0.12-10.2)$ & & & 13 & - & & \\
\hline & & PER rural & & PER urban & & SMA & & SRB urban & & THA rural & & THA urban & & TZA rural & & TZA urban \\
\hline & $\%$ & AOR $(95 \% \mathrm{Cl})$ & $\%$ & AOR $(95 \% \mathrm{Cl})$ & $\%$ & AOR $(95 \% \mathrm{Cl})$ & $\%$ & AOR $(95 \% \mathrm{Cl})$ & $\%$ & AOR $(95 \% \mathrm{Cl})$ & $\%$ & AOR $(95 \% \mathrm{Cl})$ & $\%$ & AOR $(95 \% \mathrm{Cl})$ & $\%$ & AOR $(95 \% \mathrm{Cl})$ \\
\hline Among cohabiting & & $(n=946)$ & & $(n=595)$ & & $(n=931)$ & & $(n=755)$ & & $(n=769)$ & & $(n=836)$ & & $(n=870)$ & & $(n=995)$ \\
\hline \multicolumn{17}{|c|}{ Duration of relationship } \\
\hline$>=5 \mathrm{yrs}$ & 76 & - & 69 & - & 68 & - & 74 & - & 83 & - & 74 & - & 64 & - & 63 & - \\
\hline $1-5 \mathrm{yrs}$ & 20 & $1.1(0.64-1.8)$ & 23 & $1.8(0.91-3.5)$ & 24 & $1.3(0.79-2.0)$ & 20 & $1.3(0.41-4.0)$ & 13 & $1.3(0.71-2.4)$ & 20 & $1.4(0.88-2.3)$ & 30 & $0.94(0.60-1.5)$ & 29 & $0.76(0.50-1.2$ \\
\hline$<1 \mathrm{yr}$ & 4 & $1.0(0.39-2.8)$ & 7 & $2.8(1.0-7.6)$ & 8 & $1.6(0.76-3.3)$ & 6 & $1.9(0.29-12.6)$ & 4 & $0.72(0.25-2.0)$ & 5 & $1.3(0.58-2.9)$ & 6 & $0.45(0.20-1.0)$ & 8 & $0.61(0.30-1.2$ \\
\hline Among cohabiting & & $(n=587)$ & & $(n=383)$ & & $(n=689)$ & & $(n=650)$ & & $(n=690)$ & & $(n=681)$ & & $(n=529)$ & & $(n=709)$ \\
\hline \multicolumn{17}{|l|}{ Choice of husband } \\
\hline She took part & 80 & - & 87 & - & 96 & - & 99 & - & 85 & - & 91 & - & 92 & - & 87 & \\
\hline
\end{tabular}


Table 3 Descriptive data for additional 'characteristics of union' variables, and AORs* and 95\%Cls for associations with current IPV among cohabiting and married women (Continued)

\begin{tabular}{|c|c|c|c|c|c|c|c|c|c|c|c|c|c|c|c|c|}
\hline He/family chose 2 & 20 & $0.82(0.47-1.4)$ & 13 & $0.37(0.12-1.1)$ & 4 & $0.37(0.12-1.15)$ & 1 & $135.7(6.4-2872.5)$ & 15 & $0.93(0.54-1.6)$ & 9 & $0.59(0.26-1.3)$ & 8 & $1.3(0.61-2.4)$ & 13 & $1.3(0.69-2.2)$ \\
\hline Bride price/dowry & & & & & & & & & & (binary) & & (binary) & & & & \\
\hline Dowry & & & & & & & & & 0.4 & & 1 & & 1 & $0.47(0.04-6.24)$ & 7 & $1.0(0.30-3.5)$ \\
\hline Bride price & & & & & & & & & 93 & $0.49(0.25-0.98)$ & 84 & $0.85(0.49-1.5)$ & 94 & $1.34(0.53-3.43)$ & 87 & $1.6(0.67-4.0)$ \\
\hline None & & & & & & & & & 7 & - & 16 & - & 6 & - & 5 & - \\
\hline 4 & & & & & & & & & & & & & & & & \\
\hline \multicolumn{17}{|l|}{ Polygamy } \\
\hline No & & & & & & & & & & & & & 81 & - & 82 & - \\
\hline Yes & & & & & & & & & & & & & 18 & $2.4(1.4-4.1)$ & 13 & $1.1(0.67-1.9)$ \\
\hline Don't know & & & & & & & & & & & & & 1 & & 5 & -— \\
\hline
\end{tabular}

*Adjusted for all variables in same and above sections of this Table, and for all variables in Table 2. 
mother, her partners' mother, or both (compared to no known reported abuse of either mother) being associated with increased risk of IPV in all sites (10 sites significant for women, 10 for partners, 12 for both). ORs for IPV tended to be highest where women reported that both their mothers and their partners' mothers experienced abuse (observed in 10/15 sites). Evidence from bivariate analysis in most sites showed that women who did not know whether their partners had histories of abuse were also at increased risk of IPV compared to those who reported their partners did not have these experiences.

Other experiences of violence were also associated with past year IPV, with a history of childhood sexual abuse of the woman, childhood beatings of her partner, or both consistently associated with increased risk of IPV, compared to no reports of abuse by either partner (15/15 sites for partners, all significant; 13/15 for women and both, 10 significant). Women in relationships where both she and her partner were abused in childhood are at the highest risk of IPV (true in 11/14 sites), (see Table 1). These exposure categories often contained small numbers of women. When child sexual abuse is considered in isolation at the bivariate level, its association with IPV is significant in 14/15 sites. Since CSA has also been linked to other intervening variables in the model, such as low educational attainment[22], the fact that CSA remains highly significant in the final model confirms it's importance as a risk factor for IPV.

While small numbers in the extreme exposure categories for the abuse variables result in very wide confidence intervals for some of the ORs, the consistency of 'dose-response' patterns observed for all variables in this model provides compelling evidence of the combined importance of childhood experiences of both the woman and her partner in relation to IPV in later life.

\section{Current situation model Demographics}

Younger age of women was strongly associated with increased risk of past year IPV in all sites (significant in 12). A similar pattern was seen in bivariate analysis for partner's age but this variable was excluded from multivariate models due to its strong correlation with the woman's age. In contrast, associations between IPV and an age-gap of at least 5 years between the woman and her partner were weak in most settings and the direction of the effect was context dependent. Older age of the woman was often associated with increased risk of IPV, but in only three out of fifteen sites was older age of the partner associated with increased risk of IPV. Weak associations were also seen in the other direction for age-gaps favouring either the woman or her partner.
There was some suggestion that inequality in educational level between a woman and her partner may increase her risk of experiencing IPV. This was true in nine out of 15 sites where the woman had the higher level of education ( 1 significant), and the same where her partner had the higher level. Associations tended to be weak, however, and some were also observed in the opposite direction.

There was no consistent pattern of association between IPV and relative employment status. Compared to couples in which both partners work, couples where just the man works appear to experience slightly lower levels of IPV in some settings (8/14, 2 sig). In some settings women who work when their partners do not may be at increased risk of IPV (6/14 sites, 2 significant). There is some evidence that women in relationships where neither she nor her partner work are at increased risk of IPV (8/13 sites, 2 significant). However, non-significant associations in opposite directions are also observed for these variables.

Higher socioeconomic status (SES) was associated with decreased IPV in fourteen sites (significant in 8 sites when comparing the highest status group to the lowest). This variable was more strongly associated with IPV before adjustment for other variables that may confound or mediate the effects of socioeconomic status on IPV risk.

\section{Attitudes}

In almost all sites, women who had attitudes supportive of a husband beating his wife had increased odds of IPV (13/15, 8 significant).

\section{Alcohol}

In all sites odds of IPV were higher in relationships where one or both partners had problems with alcohol, compared to relationships where neither of them did (him 14/14, 12 significant; her 10/11, 5 significant; both $11 / 11,7$ significant). In the majority of sites frequent drunkenness among men yielded higher ORs for IPV than problematic drinking by the woman (8/11), and in ten of the eleven sites ORs were higher when both had problems with alcohol.

\section{Non-partner violence}

Both a woman's experience of non-partner violence and her partner's involvement in fights with other men emerged as strong risk factors for IPV. Women's experiences of non-partner physical or sexual abuse over the age of 15 emerged as a risk-factor for IPV in almost all sites (14/15, 10 significant and 12/13, 6 significant respectively). Likewise, women whose partners were involved in a fight with another man in the past year experienced higher levels of IPV than those with partners who did not fight (significant in 13/15 sites). These factors were more strongly associated with IPV risk in the bivariate analysis. It is likely that both IPV and non- 
partner violence share common antecedents, such as CSA in the case of women, or a history of antisocial personality and alcohol abuse among men, which may account for all or part of this association [23,24]

\section{Other relationships}

Women with children from previous relationships were at increased risk of experiencing IPV in most sites (12/ 14, with 2 significant ORs). Women whose partners had had relationships with other women during their relationship also experienced higher levels of IPV than women with faithful partners (significant in 14/15 sites). Lack of knowledge/disclosure about a partner's involvement with other women was also associated with increased IPV in most sites.

\section{Characteristics of the union}

Women who were cohabiting with a partner without being formally married were at increased risk of IPV (10/12 sites, 3 significant). By contrast, women not living with their partners experienced lower levels of IPV (8/9 sites, 2 significant). There was some suggestion that those in newer relationships were at increased risk of IPV, with higher levels of IPV in relationships of less than five years compared to longer relationships, in half of the sites (mostly non-significant). There were also several sites where weak associations in the opposite direction were seen for the newest relationships (5).

A woman's participation in her choice of husband was associated with IPV differently across sites. In 6 out of 15 sites her lack of participation was associated with higher levels of IPV (3 significant), while in 8 sites it was associated with decreased IPV (1 significant).

Payment of dowry and bride price (compared to no marital exchange) was associated with IPV in some sites, though patterns of risk were difficult to interpret. In the 6 sites where dowry was paid, it was associated with higher levels of IPV in 4 sites (3 significant) and lower IPV in 1 site (not significant). Bride price was associated with decreased IPV in 4 sites (2 significant) and increased IPV in two sites (neither significant).

Women whose husbands had more than one wife were at increased risk of IPV in all 6 sites where polygamy is practised (3 statistically significant). The same was true for women who reported not knowing whether their husbands had other wives, compared to those who knew.

\section{Discussion}

Despite the wide variations in the prevalence of IPV across the study sites, many risk factors appear to affect IPV risk similarly, with secondary education, high SES, and formal marriage offering protection, and alcohol abuse, cohabitation, young age, attitudes supporting wife beating, outside sexual relationships, experiencing childhood abuse, growing up with domestic violence, and perpetrating or experiencing other forms of violence in adulthood, increasing the risk of IPV. We also found that the strength of the association was greater when both the woman and her partner had the risk or protective factor, suggesting the possibility of achieving greater prevention impact through targeting programs to couples most at risk.

Overall, our analysis demonstrates far more consistency in risk and protective factors across sites than reported by Hindin and Kishor in their analysis of violence among couples from 10 recent Demographic and Health Surveys (DHS) [12]. Among the factors they examined, only alcohol consumption by the husband and exposure to inter-parental violence were consistently associated with a woman's risk of violence in her current relationship. The WHO study explored a wider range of potential risk and protective factors and was able to exert greater control over the training of interviewers and study implementation. Research has shown that disclosure of partner violence is highly influenced by interviewer factors as well as privacy and context of the interview-factors that are more difficult to control in national surveys designed for other purposes $[16,25,26]$. For this reason, underreporting and misclassification of abuse cases may have obscured some of the associations in the DHS analysis.

Our analysis confirms that completing secondary education has a protective effect on IPV risk, whereas primary education alone fails to confer similar benefits [27]. Studies in the USA and South Africa, for example, find an inverted U-shaped relationship between IPV and education, whereby protection from IPV is seen at the lowest and highest educational levels [28,29]. Results suggesting increased protection when both women and their partners complete secondary education, and those pointing towards increased IPV risk where there is disparity in educational attainment, confirm the importance of promoting equal access to education for boys and girls, as recommended by target 4 of the Gender Equality Goal of the Millennium Development Goals.

Higher socioeconomic status is generally associated with lower levels of physical and/or sexual partner violence. Even if it is not an independent or proximate risk factor but one that is partially confounded by or mediated through other factors (as suggested by the multivariate analysis), socioeconomic status of households should be taken into account when designing and targeting IPV intervention programmes [27].

Early life experiences of abuse (including the physical abuse of boys and the sexual abuse of girls) emerge as consistently strong risk factors for IPV. In order to intervene in this inter-generational cycle of abuse, interventions must address childhood abuse and respond appropriately to children who have witnessed IPV 
against their mothers. Although the importance of the sexual abuse of children and the witnessing of marital violence by children has been documented in other studies, the potential importance of the physical abuse of boys has received less attention and merits further exploration. The consistent association between IPV and other forms of violence against women also point to the need for integrated responses to violence across sectors and programmes [30,31]. For example, programming to support children exposed to marital violence, may help reduce their risk of violence in later life.

Male behaviours commonly associated with 'traditional' masculinity [32], such as having many sexual partners, controlling female behaviour, and fighting other men, are strongly associated with IPV across all sites. Women having children from another partnership, or, in some settings, working when her partner does not, also appear to increase her risk of IPV. These results highlight the need to engage with men and women to challenge norms around what is expected of, and deemed acceptable behaviour for both men and women. Promising research from Brazil, South Africa and Uganda highlights the potential impacts on partner violence, of programmes that tackle models of masculinity and address issues of gender norms [33].

Problematic alcohol use, among both women and their partners, is consistently and strongly associated with IPV. While it is difficult to establish the temporality of the observed associations, this relationship has been repeatedly been demonstrated in studies of IPV [12,34-36]. Health services, police and addiction programmes may therefore provide important entry points to identify and refer people who may be at risk of IPV. Interventions that try to address and change cultural norms supportive of excessive alcohol use might also be expected to have knock-on effects in terms of primary violence prevention [37].

Importantly, not all variables demonstrated consistent relationships with IPV across sites, suggesting that policymakers should be cautious about any 'one model fits all' approach to IPV prevention. For example, risk associated with age disparity among partners, a woman working where her partner does not, and a woman taking an active role in choosing her partner, varies by setting. What constitutes empowerment in one setting may represent an unacceptable transgression of gender norms elsewhere. Jewkes highlights that transgression of gender norms and failure to fulfil cultural expectations of good womanhood and successful manhood are among the most important triggers for intimate partner violence [19]. She argues that what constitutes a transgression may vary by setting, thus leading to crossnational variation in the behaviours that may emerge as risk factors. The fact that we sometimes but not invariably observe increased IPV risk associated with the higher relative status of a woman (for example if she works and her partner does not) can also be interpreted in the light of theories that risk of partner violence may increase during periods of transition in gender relations. Women who step into new roles before background gender norms have shifted may be at increased risk of violence [38]. It is thus important that prevention efforts engage with both men and women [19].

\section{Strengths and Limitations}

The primary strength of our analysis is that it is based on fully comparable data from 15 culturally, economically and socially diverse sites. This type of comparison has not been possible to date in the field of IPV research, with the exception of the less tightly controlled DHS surveys. Obviously, the cross-sectional nature of this study limits the extent to which we can draw conclusions regarding temporality or the causal nature of observed associations. However, by distinguishing between early life and current characteristics, we do separate out those factors where temporality is clear from those where it is less certain.

A further limitation is that the study interviewed only women, and hence relies on women's reports of their partner's characteristics. The data on partner characteristics refers to the woman's current or most recent partner, who in some cases may not be the perpetrator of the reported violence. Since the analysis considers only past year IPV, however, the number of cases where the reported violence was perpetrated by a more distant partner is likely to be small. Any resulting misclassification would bias results towards the null rather than invalidate observed associations.

\section{Conclusions}

The multi-faceted nature of the factors that influence partner violence highlights the need for a multi-sectoral response that combines development activities, including improved access to secondary education for girls and boys, with initiatives to transform gender norms and attitudes, address prior histories of abuse, and reduce harmful drinking. Since risk of IPV is highest in younger women, schools are also an important setting for primary prevention activities, with potential to address issues of relationships, gender roles, power and coercion within existing youth violence and bullying programmes. Although there is no magic bullet to reduce partner violence, the consistency of our findings across sites suggests that a prevention strategy, once validated and refined, might have relevance in a wide range of settings. Initiatives to reduce partner violence require commitment and vision-by the international community, local governments and civil society. The time to act is now. 
As highlighted in the recent UN Campaign against violence against women-Women Won't Wait-such responses are urgently needed.

\section{Note}

${ }^{1}$ Women were defined as ever partnered if they had ever been married or lived with a partner (and therefore had been at risk of intimate partner violence). In practice, this definition varied slightly between countries in accordance with local notions of partnerships.

\section{Additional material}

\section{Additional file 1: Prevalence of physical and/or sexual intimate} partner violence among ever-partnered women, by site. Prevalence data on lifetime and past-year experience of physical and/or sexual intimate partner violence among ever-partnered women for each of the sites included in the WHO study. These data are among those core study findings previously published in the Lancet (Garcia-Moreno C, Jansen HA Ellsberg $M$, Heise $L$, Watts $C H$ : Prevalence of intimate partner violence: findings from the WHO multi-country study on women's health and domestic violence. Lancet 2006, 368(9543):1260-1269.

\section{Abbreviations}

IPV: intimate partner violence; CSA: childhood sexual abuse; DHS: Demographic and Health Survey; SES: socioeconomic status; WHO: World Health Organisation; UN: United Nations.

\section{Acknowledgements}

The study was only possible because of the dedication and hard work of all of those involved, both internationally and in the countries concerned. We would like to acknowledge and thank the more than 24000 women who participated in the study, and who gave their time to answer our questions and shared their life experiences with us. Implementation of the study involved many people in the participating institutions. We would like to acknowledge the collaborating institutions and the hard work and dedication of the country Principal Investigators: Bangladesh: Ruchira Tabassum Naved and Abbas Bhuiya (ICDDR,B: Centre for Health and Population Research, Dhaka), Safia Azim (Naripokkho, Dhaka) and Lars Ake Persson (Uppsala University, Sweden); Brazil: Lilia Blima Schraiber, Ana Flavia Lucas D'Oliveira and Ivan França-Junior (University of São Paulo, São Paulo), Carmen Simone Grilo Diniz (Feminist Collective for Health and Sexuality, São Paulo), Ana Paula Portella (SOS Corpo Genero e Cidadania, Recife), Ana Bernarda Ludermir (Federal University of Pernambuco, Recife); Ethiopia: Yemane Berhane, Negussie Deyessa, Yegomawork Goyasse, Atalay Alem, Derege Kebede and Alemayehu Negash (Addis Ababa University, Addis Ababa), Ulf Hogberg, Gunnar Kullgren and Maria Emmelin (Umeå University, Sweden), Mary Ellsberg (PATH, Washington, DC, USA); Japan: Mieko Yoshihama (University of Michigan, Ann Arbor, USA), Saori Kamano (National Institute of Population and Social Security Research, Tokyo), Hiroko Akiyama (University of Tokyo, Tokyo), Fumi Hayashi (Toya Eiwa University, Tokyo), Tamie Kaino (Ochanomizu University, Tokyo), Tomoko Yunomae (Japan Accountability Caucus, Beijing, Tokyo); Namibia: Eveline January, Hetty RoseJunius and Johan Van Wyk (Ministry of Health and Social Services, Windhoek), Alvis Weerasinghe (National Planning Commission, Windhoek); Peru: Ana Güezmes García (Centro de la Mujer Flora Tristan, Lima), Nancy Palomino Ramirez and Miguel Ramos Padilla (Universidad Peruana Cayetano Heredia, Lima); Samoa Tina Tauasosi-Posiulai, Tima Levai-Peteru, Dorothy Counts and Chris McMurray (Secretariat of the Pacific Community); Serbia and Montenegro: Stanislava Otaševic and Silvia Koso (Autonomous Women's Center Against Sexual Violence, Belgrade), Viktorija Cucic (University of Belgrade, Belgrade); Thailand: Churnrurtai Kanchanachitra, Kritaya Archavanitkul and Wassana Im-em (Mahidol University, Bangkok), Usa Lerdsrisanthat (Foundation for Women, Bangkok); The United Republic of Tanzania: Jessie Mbwambo and Gideon Kwesigabo (Muhimbili College of Medical Sciences), Joe Lugalla (University of New Hampshire, Durham, USA),
Sherbanu Kassim (Women's Research and Documentation Project, Dar es Salaam).We also gratefully acknowledge the interviewers and other office and field staff in the countries, who all worked with immense dedication and commitment to ensure the successful implementation of the study. The study was supported with funding provided to WHO from the Governments of the Netherlands, Norway, Sweden, Switzerland, and the UK, and the Rockefeller Foundation (who also provided the use of their meeting place in Bellagio in May, 2004, for a meeting of the WHO VAW Study team). $\mathrm{WHO}$ provided the funding for the implementation of the study in 6 of the 8 initial countries: Brazil, Japan, Namibia, Peru, Thailand, and the United Republic of Tanzania, with some of these countries receiving additional funds from local sources. In Bangladesh, the study was funded by the Urban Primary Health Care project of the Government of Bangladesh; in Ethiopia by the Swedish Agency for Research Cooperation with Developing Countries (SAREC/Sida); in Samoa, by the United Nations Fund for Population Activities (UNFPA); and in Serbia and Montenegro, by Trocaire.

\section{Author details}

${ }^{1}$ London School of Hygiene and Tropical Medicine, 15-17 Tavistock Place, London WC1H 9SH, UK. 'WHO, Avenue Appia 20, 1211 Geneva 27, Switzerland. ${ }^{3}$ International Centre for Research on Women, 1120 20thSt NW, Suite 500 North, Washington D.C. 20036, USA. ${ }^{4}$ Geneva, Switzerland.

\section{Authors' contributions}

CG-M, HAFMJ, ME, LH and CHW all participated in the study design and implementation. CG-M was the study coordinator. HAFMJ set up and supported data collection and processing in the countries and managed the central database. TA carried out the statistical analysis for this paper and drafted the manuscript. LH and CW helped draft the manuscript. KD and LK provided support with the statistical analysis and helped draft the manuscript. CG-M reviewed a draft of the manuscript. All authors read and approved the final manuscript.

\section{Competing interests}

The authors declare that they have no competing interests.

Received: 18 October 2010 Accepted: 16 February 2011

Published: 16 February 2011

\section{References}

1. Garcia-Moreno C, Jansen HA, Ellsberg M, Heise L, Watts CH: Prevalence of intimate partner violence: findings from the WHO multi-country study on women's health and domestic violence. Lancet 2006, 368(9543):1260-1269.

2. Mayhew S, Watts C: Global rhetoric and individual realities: linking violence against women and reproductive health. In Health policy in a globalising world. Edited by: Lee K, Buse K, S F. Cambridge: Cambridge University Press; 2002:159-180.

3. Campbell J, Jones AS, Dienemann J, Kub J, Schollenberger J, O'Campo P, Gielen AC, Wynne C: Intimate partner violence and physical health consequences. Arch Intern Med 2002, 162(10):1157-1163.

4. Ellsberg M, Jansen HA, Heise L, Watts CH, Garcia-Moreno C: Intimate partner violence and women's physical and mental health in the WHO multi-country study on women's health and domestic violence: an observational study. Lancet 2008, 371(9619):1165-1172.

5. Maman S, Campbell J, Sweat MD, Gielen AC: The intersections of HIV and violence: directions for future research and interventions. Soc Sci Med 2000, 50(4):459-478.

6. Garcia-Moreno C, Watts C: Violence against women: its importance for HIV/AIDS. AIDS 2000, 14(Suppl 3):S253-265.

7. Jewkes RK, Levin JB, Penn-Kekana LA: Gender inequalities, intimate partner violence and HIV preventive practices: findings of a South African cross-sectional study. Soc Sci Med 2003, 56(1):125-134.

8. Dunkle KL, Jewkes RK, Brown HC, Gray GE, Mclntryre JA, Harlow SD: Gender-based violence, relationship power, and risk of HIV infection in women attending antenatal clinics in South Africa. Lancet 2004, 363(9419):1415-1421.

9. Koenig MA, Stephenson R, Ahmed S, Jejeebhoy SJ, Campbell J: Individual and contextual determinants of domestic violence in North India. American Journal of Public Health 2006, 96(1):1-7.

10. Naved RT, Persson LA: Factors associated with spousal physical violence against women in Bangladesh. Studies in Family Planning 2005, 36(4):289-300. 
11. Parish WL, Wang T, Laumann EO, Pan S, Luo Y: Intimate partner violence in China: National prevalence, risk factors and associated health problems. International Family Planning Perspectives 2004, 30(4):174-181.

12. Hindin $M$, Kishor $S$, Ansara D: Intimate partner violence among couples in 10 DHS countries: Predictors and health outcomes. DHS Analytical Studies No 18 Calverton, MD: Macro International; 2008.

13. Stith S, Smith D, Penn C, Ward D, Tritt D: Intimate partner physical abuse perpetration and victimization risk factors: A meta-analytic review. Aggression and Violent Behavior 2004, 10(1):65-98.

14. Koenig MA, Ahmed S, Hossain MB, Khorshed Alam Mozumder AB: Women's status and domestic violence in rural Bangladesh: individualand community-level effects. Demography 2003, 40(2):269-288.

15. Kishor S, Johnson K: Profiling domestic violence: A multicountry study. Calverton, Maryland, USA: ORC Macro; 2004.

16. Ellsberg M, Heise L, Pena R, Agurto S, Winkvist A: Researching domestic violence against women: methodological and ethical considerations. Stud Fam Plann 2001, 32(1):1-16.

17. Heise LL: Violence against women: an integrated, ecological framework. Violence Against Women 1998, 4(3):262-290.

18. Urbina: Conflict within intimacy a socio-demographic analysis of male involvement in physical intimate partner violence in Mexico. 2005.

19. Jewkes R: Intimate partner violence: causes and prevention. Lancet 2002, 359(9315):1423-1429.

20. WHO: Putting women's safety first: ethical and safety recommendations for research on domestic violence against women. Geneva: World Health Organization; 1999.

21. Hox J: Multilevel analysis: techniques and applications New Jersey: Lawrence Erlbaum Associates; 2002.

22. Messman-Moore TL, Long PJ: The role of childhood sexual abuse sequelae in the sexual revictimization of women - An empirical review and theoretical reformulation. Clin Psychol Rev 2003, 23(4):537-571.

23. Fargo JD: Pathways to adult sexual revictimization: direct and indirect behavioral risk factors across the lifespan. I Interpers Violence 2009, 24(11):1771-1791.

24. Capaldi D, Kim H, Pears K: The association of partner violence to child maltreatment: A common conceptual framework. In Preventing Partner Violence: Research and Evidence-based Intervention Strategies. Edited by: Whitaker DK, Lutzker J. Washington DC: American Psychological Association; 2009:

25. Andersson N, Cockcroft A, Ansari N, Omer K, Chaudhry UU, Khan A, Pearson $L$ : Collecting reliable information about violence against women safely in household interviews: experience from a large-scale national survey in South Asia. Violence Against Women 2009, 15(4):482-496.

26. Jansen HAFM, Watts C, Ellsberg M, Heise L, Garcia-Moreno C: Interviewer training in the WHO Multi-country Study on Women's Health and Domestic Violence. Violence against Women 2004, 10(7):831-849.

27. Vyas $S$, Watts $C$ : How does economic empowerment affect women's risk of intimate partner violence in low and middle income country settings?: A systematic review of published evidence. J Int Dev 2009, 21:577-602.

28. Straus M, Gelles R, Steinmetz S: Behind closed doors: violence in the American family New York: Anchor Press; 1980.

29. DoH: South Africa Demographic and Health Survey 1998: final report. Pretoria: Department of Health; 2002.

30. Harvey A, Garcia Moreno C, Butchart A: Primary prevention of intimate partner violence and sexual violence: Background paper for WHO expert meeting May 2-3, 2007. Geneva: World Health Organization, Department of Violence and Injuy Prevention and Disability; 2007.

31. Krug $E G$, Mercy JA, Dahlberg $L L, Z$ wi $A B$ : The world report on violence and health. Lancet 2002, 360(9339):1083-1088.

32. Barker $G$, Ricardo $C$ : Young men and the construction of masculinity in sub-saharan africa: Implications for HIV/AIDS, conflict and violence. Conflict prevention and reconstruction Washington D.C.: The World Bank; 2005.

33. Barker G, Ricardo C, Nascimento M, Olukoya A, Santos C: Questioning gender norms with men to improve health outcomes: Evidence of impact. Global Public Health: An International Journal for Research, Policy and Practice 2010, 5:539-553.

34. Foran HM, O'Leary KD: Alcohol and intimate partner violence: a metaanalytic review. Clin Psychol Rev 2008, 28(7):1222-1234.
35. Gil-Gonzalez D, Vives-Cases C, Alvarez-Dardet C, Latour-Perez J: Alcohol and intimate partner violence: do we have enough information to act? Eur J Public Health 2006, 16(3):278-284.

36. Leonard KE: Alcohol and intimate partner violence: When can we say that heavy drinking is a contributing cause of violence? Addiction 2005, 100:422-425.

37. Room R: Alcohol in developing societies: A public health approach Geneva: World Health Organization; 2003.

38. Counts D, Brown J, Campbell J: Sanctions and Sanctuary Boulder: Westview Press; 1992.

\section{Pre-publication history}

The pre-publication history for this paper can be accessed here: http://www.biomedcentral.com/1471-2458/11/109/prepub

\section{doi:10.1186/1471-2458-11-109}

Cite this article as: Abramsky et al.: What factors are associated with recent intimate partner violence? findings from the WHO multi-country study on women's health and domestic violence. BMC Public Health 2011 11:109.

\section{Submit your next manuscript to BioMed Central and take full advantage of:}

- Convenient online submission

- Thorough peer review

- No space constraints or color figure charges

- Immediate publication on acceptance

- Inclusion in PubMed, CAS, Scopus and Google Scholar

- Research which is freely available for redistribution

Submit your manuscript at www.biomedcentral.com/submit
Ciomed Central 